ISSN 0103-5150

Fisioter. Mov., Curitiba, v. 24, n. 1, p. 75-85, jan./mar. 2011 Licenciado sob uma Licença Creative Commons

\title{
Influência do fortalecimento abdominal na função perineal, associado ou não à orientação de contração do assoalho pélvico, em nulíparas
}

\author{
The abdominal strengthen influence in perineal function when \\ associated, or not, to the orientation of the pelvic floor contraction, \\ in nulliparous
}

\author{
Raciele Ivandra Guarda Korelo ${ }^{[a]}$, Célia Regina Kosiba ${ }^{[b]}$, Letícia Grecco ${ }^{[c]}$, Rafaela Abreu Matos ${ }^{[d]}$ \\ [a] Fisioterapeuta e Mestre em Tecnologia em Saúde pela Pontifícia Universidade Católica do Paraná (PUCPR), \\ docente do curso de Fisioterapia da Faculdade Dom Bosco e da Faculdade Evangélica do Paraná, e-mail: \\ racieleguarda@dombosco.com.br \\ [b] Fisioterapeuta graduada pela Faculdade Dom Bosco, Curitiba, PR - Brasil, e-mail: celiakosiba@yahoo.com.br \\ [c] Fisioterapeuta graduada pela Faculdade Dom Bosco, Curitiba, PR - Brasil, e-mail: legrecco@yahoo.com.br \\ [d] Fisioterapeuta graduada pela Faculdade Dom Bosco, Curitiba, PR - Brasil, e-mail: rafaela.abreu@yahoo.com.br
}

\section{Resumo}

Objetivo: Verificar se o fortalecimento abdominal promove influência na musculatura do assoalho pélvico feminino. Materiais e métodos: Caracterizou-se como um ensaio clínico, realizado com 21 nulíparas, com idade média de 21,7 anos, divididas por conveniência em dois grupos: A ( $n=10)$ - com orientação de contração perineal simultânea à contração abdominal e B $(n=11)$ - sem orientação de contração perineal. As participantes foram submetidas à avaliação ginecológica e abdominal pré e pós-intervenção por meio de: avaliação funcional do assoalho pélvico (AFA), cones vaginais, perineometria, teste de flexão anterior de tronco, descida de membros inferiores, força de oblíquos e endurance abdominal. o protocolo de fortalecimento abdominal continha três exercícios distintos, associados ou não à contração perineal, com três séries de dez repetições e repouso de 60 segundos, três vezes por semana, em um período de seis semanas. Para a análise estatística foi aplicado o teste t de Student e ANOVA. Resultados: A comparação entre os valores iniciais e finais demonstrou diferenças significativas entre os dois grupos na avaliação do períneo, somente no teste com cones $(p=0,00)$ e na perineometria (resistência/pressão) $(p=0,03)$, indicando piora das variáveis no grupo B. Já na avaliação abdominal, o grupo A apresentou resultado significativo em três testes (descida de membros inferiores: $p=0,03$, endurance: $p=0,03$ e endurance modificado: $p=0,00$ ) comparando-se a somente um do grupo B (endurance: $\mathrm{p}=0,01$ ). Conclusões: Sugere-se que a associação de exercícios 
de fortalecimento abdominal com o perineal pode potencializar o ganho de força e a função desses grupos musculares, e a falta dessa associação pode prejudicar a funcionalidade do assoalho pélvico.

Palavras-chave: Assoalho pélvico. Períneo. Exame físico. Músculos abdominais e fisioterapia.

\section{Abstract}

Objective: Verify if abdominal strengthen has influence in pelvic floor muscles. Materials and methods: This work consisted on a clinical trial approach twenty-one nulliparous, with average ages of 21,7 years old, divided in two groups: $A(n=10)$ with perineal contraction orientation simultaneously to abdominal contraction and $B(n=11)$ with no perineal contraction orientation. The participants were submitted to a gynecological and abdominal assessment pre and post intervention through: pelvic floor functional evaluation, vaginal cones, perineometry, trunk anterior flexion test, inferiors members descent, force of oblique and abdominal endurance. The abdominal strength protocol contained three distinct exercises, associated or not to the pelvic floor contraction, with three series of ten repetitions and sixty seconds of interval, three times a week over a period of six weeks. The Student's t-test and the ANOVA were used for the statistical analysis. Results: The comparison between initial and final values showed significant differences between the two groups in the assessment of the perineum, the test only with cones ( $p=0.00)$ and perineometry (strength/pressure) $(p=0.03)$, indicating a worsening of the variables in group B. Already in abdominal evaluation, group A showed significant results in three trials (the inferiors members descent: $p=0.03$, abdominal endurance: $p=0.03$ and modified endurance: $p=0.00$ ) compared to only one group B (addominal endurance: $p=0.01$ ). Conclusions: Suggest that the combination of abdominal strengthening exercises with perineal may boost the gain in strength and function of these muscle groups and the lack of association may impair the functionality of the pelvic floor.

Keywords: Pelvic floor. Perineum. Physical examination. Abdominal muscles and physiotherapy.

\section{Introdução}

A disfunção do assoalho pélvico (AP) feminino é a condição clínica que acomete um número crescente de mulheres a cada ano, tendo como consequência a incontinência urinária (IU), a incontinência fecal, a incontinência de flatos, as distopias genitais, as anormalidades do trato urinário inferior, as disfunções sexuais, a dor pélvica crônica e/ou os problemas menstruais (1).

A incontinência urinária compreendida como uma patologia, segundo a International Continence Society, é definida como a perda involuntária de urina e classificada em três tipos principais: esforço, urgência e mista; sendo a mais comum a de esforço, determinada por fraqueza muscular, atingindo em número maior o sexo feminino (2).

A incontinência é um problema que afeta mundialmente milhares de mulheres em idade ativa. Cerca de 200 milhões de pessoas no mundo apresentam algum tipo de IU e acredita-se que um quarto das mulheres entre 30 e 59 anos já vivenciou algum episódio relacionado a esse problema. Em estudo epidemiológico no Brasil, foi encontrada a prevalência de $35 \%$ de queixa de perda urinária aos esforços em 456 mulheres entre 45 e 60 anos de idade (2). A queixa em mulheres atletas é de $22 \%$ a $47 \%$, sofrendo variação de acordo com a atividade (3). As causas ainda não estão completamente elucidadas e algumas ainda são pouco discutidas (2).

Visto que a IU não se limita apenas aos seus aspectos físicos, ela se tornou um problema social que está diminuindo a qualidade de vida (QV) das cidadãs que sofrem desse mal, por ocasionar diminuição de contatos sexuais, ocupacionais e pessoais, sintomas depressivos e diminuição da autoestima, levando ao isolamento como consequência do constrangimento pela perda involuntária de urina $(2,4,5)$.

A IU de esforço atinge com mais frequência mulheres entre 25 e 49 anos de idade. A atividade física de alto impacto é um fator de risco para desenvolvê-la, sendo percebida apenas a partir da realização de atividades que predisponham a perda de urina. Outros fatores de risco incluem constipação, tosse crônica do fumante ou doença pulmonar, obesidade e ocupações que exigem levantamento crônico de peso (1). 
Foi somente no início dos anos 80 que se relatou a preocupação com a prevalência e a incidência da IU em atletas. No entanto, existem poucas publicações científicas sobre o assunto e, principalmente, estudos que comprovem qual tipo de atividade física pode influenciar na função perineal.

Os exercícios abdominais aumentam a pressão intra-abdominal (PIA), comprimindo vísceras e distribuindo a carga para o AP, e os aumentos na PIA afetam indiretamente a pressão sobre a bexiga urinária. Esse aumento da PIA é condição favorável para haver perda involuntária de urina em ocasiões em que as respostas da musculatura do assoalho pélvico (MAP) se encontram alteradas (1).

A contração da musculatura abdominal ocorre simultaneamente à contração do $\mathrm{AP}$, demonstrando ação sinérgica abdomino-pélvica de maneira voluntária ou não (1). A maioria das atividades físicas não envolve contração voluntária desses músculos durante a realização de exercícios que aumentem a PIA (2).

Pouco se sabe acerca do funcionamento dos músculos do períneo durante a prática de exercícios físicos. Portanto, mulheres que fazem exercícios sem realizar contração perineal não possuem, necessariamente, o AP forte e podem evidenciar a IU (1).

Estudos que quantificam a influência de protocolos de fortalecimento abdominal sobre a força da MAP são escassos. 0 objetivo do presente estudo foi verificar, por meio de um protocolo de fortalecimento abdominal, se a funcionalidade da MAP é alterada. Para tanto, foram comparados dois grupos com ou sem orientação de associar a contração do períneo simultaneamente ao exercício abdominal.

\section{Materiais e métodos}

0 presente trabalho caracteriza-se como um ensaio clínico. A população foi composta por 21 mulheres nulíparas, acadêmicas, com média de idade de $21,75( \pm 4,14)$ anos. Os critérios de exclusão foram: praticantes de exercícios que envolviam a musculatura abdominal; mulheres que realizaram qualquer tipo de cirurgia abdominal e pélvica há menos de 6 meses; portadoras de incontinência urinária; gestantes; mulheres que nunca tiveram relação sexual; portadoras de doenças osteomioarticulares e/ou neurológicas em níveis que atinjam inervações de abdominais e musculatura perineal (entre L1 e S4); mulheres com IMC acima de 27; que não atingissem o grau 3 nos testes de força de abdominais ou não apresentassem integridade ao teste de sensibilidade do assoalho pélvico.

0 presente estudo obedeceu às normas da Resolução n. 196/96, que dispõe sobre as diretrizes para pesquisas envolvendo seres humanos, e foi aprovado pelo Comitê de Ética em Pesquisa da instituição sob o protocolo n. 0003.0.301.000-09. Os indivíduos foram esclarecidos sobre os detalhes do estudo, sendo obtidos seus consentimentos informados por escrito por meio da assinatura do termo de consentimento livre e esclarecido. Depois de informados, os indivíduos foram avaliados com registro na ficha de avaliação, onde constavam os dados de identificação, a anamnese, o exame físico e a avaliação de força abdominal e perineal.

A avaliação perineal foi realizada somente após treino proprioceptivo (6) para evitar interferências em razão da falta de percepção da contração perineal e consistiu em avaliação funcional do assoalho pélvico (AFA) pela palpação bidigital (7), avaliação passiva de cones vaginais (7) e perineometria realizada com o miofeedback Perina ${ }^{\circledR}$ 9962 - cedido pelo fabricante Quark -, o qual mensura a pressão de 0 a $50 \mathrm{mmHg}$ e possibilita registrar o tempo de contração máxima do assoalho pélvico, tanto de forma rápida como sustentada, exercida pelo sujeito de acordo com a metodologia descrita por Moreno (8).

Os testes para avaliação da força abdominal das participantes incluiu: teste de flexão anterior de tronco (9), teste de abaixamento de membros inferiores (9), teste para graduação da força muscular dos oblíquos (10) e teste de endurance do rolinho modificado para abdominais (11) para verificar o desempenho dessa musculatura e constatar quantas repetições o indivíduo consegue completar, mantendo o mesmo tempo de descida e subida da primeira contração, e realizando o mesmo grau de amplitude em todas as repetições.

Após as avaliações, as voluntárias foram divididas por conveniência em dois grupos: A e B, seguindo a ordem de inclusão no estudo. A primeira participou do grupo A, a segunda do B, a terceira do A e assim sucessivamente, porém, caso as mulheres da pesquisa tivessem laços de amizade ficariam no mesmo grupo, para evitar que comentassem sobre a forma como a técnica estava sendo aplicada, o que seria diferente para cada uma. 0 grupo A 
compreendeu as mulheres que realizaram fortalecimento abdominal com instruções para contrair o períneo durante a realização do exercício, já o grupo $B$ não recebeu essas orientações.

0 protocolo de fortalecimento abdominal (Apêndice 1) teve duração de seis semanas, com atividades realizadas três vezes por semana. Este consistiu em três exercícios distintos (flexão anterior de tronco, descida de membros inferiores e fortalecimento de oblíquos) normalmente realizados em academias e baseados na orientação biomecânica dos testes utilizados na avaliação $(9,10)$. Os exercícios foram realizados em três séries de dez repetições, intervaladas com tempo de repouso de 60 segundos.

Ao término do protocolo de intervenção, as voluntárias foram submetidas aos mesmos testes de avaliação para a comparação dos resultados, sendo a análise estatística realizada pelo teste $t$ de Student e ANOVA, para os quais foi adotado um nível de significância de $5 \%$.

\section{Resultados}

A amostra foi composta por 21 voluntárias, nulíparas e sexualmente ativas. As características da amostra, separadas por grupos, podem ser visualizadas na Tabela 1. Já os resultados pré e pós-intervenção estão apresentados na Tabela 2 .

A comparação entre os valores iniciais e finais demonstrou diferenças significativas entre os dois grupos na avaliação do períneo, somente no teste com cones $(p=0,00)$ e na perineometria (resistência/pressão) $(p=0,03)$, sugerindo piora na maioria das variáveis no grupo $B$, apesar de não serem estatisticamente significantes.

Já na avaliação abdominal, o grupo A apresentou resultado significativo em três testes (descida de membros inferiores: $p=0,03$, endurance rolinhos completos: $p=0,03$ e endurance rolinhos modificados: $p=0,00)$, comparando-se a somente um do grupo B (endurance rolinhos completos: $\mathrm{p}=0,01$ ).

Tabela $\mathbf{1}$ - Características da amostra

\begin{tabular}{lcc}
\hline Características & Grupo A & Grupo B \\
\hline Idade (média, desvio-padrão) & $23,90 \pm 4,51$ & $19,6 \pm 2,37$ \\
IMC (média, desvio-padrão) & $21,18 \pm 1,91$ & $21,75 \pm 1,33$ \\
Atividade física & & \\
$\quad$ Já praticou atividade física & $9(90 \%)$ & $8(73 \%)$ \\
Média da duração da atividade (meses) & 34,22 & 14,75 \\
Frequência (dias/semana) & 3,55 & 3,5 \\
Há quanto tempo parou & 21,77 & 12,65 \\
Modalidades & & \\
$\quad$ Musculação & $4(44 \%)$ & $2(25 \%)$ \\
$\quad$ Lutas & $2(22 \%)$ & $2(25 \%)$ \\
$\quad$ Ginástica & $1(11 \%)$ & $3(38 \%)$ \\
$\quad$ Dança do ventre & $1(11 \%)$ & $0(0 \%)$ \\
$\quad$ Futebol & $1(11 \%)$ & $0(0 \%)$ \\
$\quad$ Vôlei & $0(0 \%)$ & $1(13 \%)$ \\
Fumantes & $1(10 \%)$ & $1(9 \%)$ \\
$\quad$ Número de cigarros por dia & 3 & 1 \\
Consome café & $3(30 \%)$ & $7(64 \%)$ \\
$\quad$ Quantidade de café (xícaras/dia) & 3 & 1 \\
Frequência urinária maior que 8 vezes/dia & 1 & 1 \\
\hline
\end{tabular}


Tabela 2 - Resultados encontrados no estudo entre os grupos

\begin{tabular}{|c|c|c|c|}
\hline Variáveis de avaliação & $\begin{array}{c}\text { Grupo A } \\
\text { inicial-final ( } p \text {-valor) }\end{array}$ & $\begin{array}{c}\text { Grupo B } \\
\text { inicial-final ( } p \text {-valor) }\end{array}$ & $\begin{array}{c}\mathrm{p} \text {-valor } \\
\text { entre os dois grupos }\end{array}$ \\
\hline \multicolumn{4}{|l|}{ Períneo } \\
\hline AFA & $4,6-4,7(0.34)$ & $5,0-5,0(1.00)$ & 1.00 \\
\hline Cones vaginais & $3,7-4,2(0.17)$ & $4,5-4,6(0.34)$ & $0.00^{*}$ \\
\hline \multicolumn{4}{|l|}{ Perineometria } \\
\hline Valor da contração rápida (mmHg) & $15,88-19,82(0.34)$ & $25,97-22,05^{\star \star}(0.30)$ & 0.15 \\
\hline \multicolumn{4}{|l|}{ Contração prolongada } \\
\hline Tempo de sustentação (seg) & $24,33-25,54(0,72)$ & $26,27-25,13^{* *}(0.66)$ & 0.78 \\
\hline Número de repetições & $4,3-4,1^{\star \star}(0.48)$ & $5,7-3,7^{\star *}(0.18)$ & 0.30 \\
\hline Pressão (mmHg) & $16,1-20,8(0.10)$ & $26,1-25,65^{\star *}(0,91)$ & 0.31 \\
\hline \multicolumn{4}{|l|}{ Resistência } \\
\hline Número de repetições & $7,8-12,0(0.38)$ & $10,1-10,3(0.97)$ & 0.62 \\
\hline Pressão (mmHg) & $13,6-19,4(0.25)$ & $26,7-20,35^{\star \star}(0.02)^{\star}$ & $0.03^{*}$ \\
\hline \multicolumn{4}{|l|}{ Abdômen } \\
\hline Teste de flexão anterior & $4,45-4,7(0.34)$ & $5,0-5,0(1.00)$ & 0.33 \\
\hline Descida de membros inferiores & $3,33-3,48(0.03)^{*}$ & $3,36-3,6(0.06)$ & 0.25 \\
\hline Força de oblíquos & $3,8-4,05(0.29)$ & $4,85-4,9(0.72)$ & 0.46 \\
\hline Endurance (rolinhos completos) & $39,3-95,4(0.03)^{*}$ & $30,3-52,3(0.01)^{*}$ & 0.18 \\
\hline Endurance (rolinhos modificados) & $2,6-13,3(0.00)^{*}$ & $4,1-3,9 * *(0.94)$ & $0.01^{*}$ \\
\hline
\end{tabular}

Legenda: * = dados com significância estatística; ** = resultado do teste com diminuição na variável avaliada, indicando piora.

\section{Discussão}

A perda involuntária de urina associada à fraqueza do assoalho pélvico diminui a qualidade de vida das mulheres, visto que interfere nos fatores: físico, social, psicológico, ocupacional, doméstico, sexual (12). Vários são os achados predisponentes para a IU de esforço, sendo a idade um dos principais, cuja incidência em geral é de $43 \%$ após os 35 anos, fato desencadeado normalmente a partir do climatério, geralmente relacionado ao hipoestrogenismo (12-17).

Não há relação entre a IU e irregularidades menstruais (8), porém, em alguns casos ela pode ocorrer com o início do hipoestrogenismo, o que diminui a capacidade vesical de 500 a $600 \mathrm{~mL}$ para 250 a $300 \mathrm{~mL}$, fato que pode ocorrer também na presença de doenças crônicas e aumento do IMC [peso (kg)/ estatura $\left.(\mathrm{m})^{2}\right]$. O IMC é classificado em: baixo peso: IMC $<22 \mathrm{~kg} / \mathrm{m}^{2}$, eutrofia: IMC entre 22 e $27 \mathrm{~kg} / \mathrm{m}^{2}$ e excesso de peso/obesidade: $\mathrm{IMC}>27 \mathrm{~kg} / \mathrm{m}^{2}$ (18).
A redução da capacidade vesical é determinante para o aumento da frequência urinária. 0 aumento do peso eleva a PIA, outro fator causal. Por esse motivo, o presente estudo contemplou mulheres entre 18 e 35 anos de idade, cuja média foi de $21,75 \pm$ 4,14 , com IMC menor ou igual a $27 \mathrm{~kg} / \mathrm{m}^{2}$, com média de $21,47 \mathrm{~kg} / \mathrm{m}^{2} \pm 1,63$, e excluiu portadoras de doenças osteomioarticulares.

A histerectomia, a excisão ou o prolapso do útero comprometem as funções do AP por alterarem as estruturas de sustentação da bexiga e da uretra. Além disso, a gestação e o peso do feto também alteram a PIA e a pressão intravesical (PIV) e, durante a passagem do bebê pelo canal vaginal, pode haver lesão do AP (13). Um estudo realizado em mulheres com seis meses pós-parto demonstrou que a prevalência de perda de urina foi maior naquelas que sofreram de IU durante a gravidez (19). Trinta por cento das mulheres que praticam atividade física queixam-se de perdas urinárias (20) e a incidência varia de $28 \%$ 
a 51\% em mulheres atletas, dançarinas e militares, com idade média de 19,9 a 31,8 anos (21). De acordo com essas informações, participaram da presente pesquisa somente nulíparas e não praticantes de exercícios abdominais extras.

O cigarro provoca queda nos níveis de estrógeno, antecipando a menopausa e aumentando a frequência e a intensidade da tosse, o que desencadeia aumento da PIV. A ação da cafeína aumenta a atividade vesical por ter efeito excitante sob a musculatura do detrusor, aumentando o volume urinário, embora estudos não revelem se a diminuição da ingestão de café reduz os sintomas (13).

0 exercício físico intenso é um fator que potencializa a prevalência de IU em mulheres jovens e nulíparas, principalmente envolvendo esportes que exijam trabalho intenso da musculatura abdominal, aumentando a PIA e afetando de forma indireta a pressão sobre a bexiga urinária, como é o caso das ginastas $(1-3,13)$. Os achados determinam que a maioria das atividades físicas não envolve contração voluntária da MAP, o que pode acarretar em deficiência funcional por perda ou ausência da consciência e coordenação das estruturas neuromusculares do $\mathrm{AP}$, levando à hipotrofia por desuso $(2,8,22)$.

0 descondicionamento e a carga repetida sobre a MAP, concomitante ao aumento frequente da PIA, diminuem a eficiência mecânica do AP e alteram a composição de alguns músculos. Portanto, mulheres que fazem exercícios físicos não possuem necessariamente músculos perineais mais fortes do que as que não fazem $(2,23)$.

Por meio da AFA, a qual recruta predominantemente fibras fásicas, cujo tempo de contração foi de cinco segundos, o grupo A teve um incremento no seu grau de força e o B manteve-se igual, valores esses não significativos nem inter nem entre os grupos. As voluntárias que realizaram a contração perineal durante o treinamento abdominal apresentaram aumento da força. Elas contraíram a MAP por nove segundos em cada repetição de exercício abdominal. 0 ganho de força se justifica pelas fibras do tipo II possuírem rápida velocidade de contração e baixa resistência à fadiga, sendo responsáveis pela força (24-26).

No teste dos cones, o qual avalia a musculatura tônica do AP, o grau do A e do B aumentou, porém, sem significância. Ambos evoluíram, principalmente o grupo A em relação ao B, uma vez que mostrou diferença significativa. Essa diferença pode ser justificada porque o exercício de descida de MMII do grupo A exigia contração perineal durante todas as repetições de cada série, totalizando 80 segundos de contração. Esse tipo de fibra possui a característica de resposta imediata a mudanças súbitas de pressão, o que no AP faz com que a continência seja mantida. A musculatura tônica perde linearmente suas fibras, fato que pode ser prevenido por meio de exercícios (15).

Para a avaliação da contração rápida na perineometria, o grupo A aumentou e o B regrediu, apesar de estatisticamente isso não ser significante nem entre nem inter grupos. No treino de força, a proporção de fibras musculares tipo II cresce significativamente, aumentando assim a força muscular (27, 28), fato observado também na presente pesquisa.

No teste de contração prolongada, o grupo A diminuiu as repetições e a pressão aumentou assim como o tempo de sustentação. Tais diferenças não foram significantes, assim como houve redução nas três variáveis avaliadas (repetições, pressão e tempo) do grupo B, sugerindo piora. Também não houve significância entre os grupos. 0 limiar de continência se dá pela quantidade e pelo tempo que os músculos do períneo suportam esforços e impactos repetitivos. Ao atingir esse limiar, os músculos fadigados perdem sua capacidade, principalmente se não estiverem condicionados (2). No primeiro momento, os músculos perineais do grupo A não foram treinados, porém, após o condicionamento aumentou o tempo de sustentação da contração.

Apesar do aumento do número de contrações no grupo $A$ e no $B$, isso não foi significante nem antes e nem depois, bem como entre os grupos. A primeira pressão do grupo A aumentou e não demonstrou significância, mas a pressão do grupo B diminuiu significativamente, demonstrando significância entre os grupos. A elevação da pressão ao nível do AP acontece antes do aumento da PIA, o que indica que a ativação dos músculos do períneo acontece por mecanismo central, casualmente regulado pela vontade, não necessariamente em resposta ao aumento da PIA. Estudos demonstraram a importância do treino da MAP concomitantemente ao treino abdominal, realizando a contração voluntária daquela musculatura antes do trabalho abdominal (14).

Observando os testes que envolviam a pressão perineal, o grupo B sofreu redução, mesmo que não significativa; em contrapartida, o grupo A obteve aumento da pressão em todos os testes. Isso ocorre 
em razão do sinergismo entre a musculatura do AP e abdominal (1).

Os distúrbios urinários, causados pela insuficiência da MAP, ocorrem por conta da hiperpressão abdominal. Esta é compensada por uma contrapressão do AP. No momento do aumento da PIA, os órgãos pélvicos são empurrados para o diafragma urogenital, o qual responde com uma contração para suportar essa pressão e sustentar os órgãos. A mulher que apresenta um abdominal forte reage a essa força para baixo que refletirá diretamente sobre o AP (3).

Quando comparadas as médias dos testes de força e resistência abdominal, o de flexão anterior de tronco obtido no grupo A aumentou e o B manteve grau máximo, ainda que não tenha sido significativo nem inter nem entre os grupos.

Na descida de membros inferiores, o grupo A aumentou seu grau, havendo significância. 0 grupo $B$ aumentou, mas sem significância, bem como entre os grupos. Na avaliação da força dos oblíquos, ambos aumentaram seu grau, sem significância. No teste de endurance não foi significante o aumento do número de rolinhos completos entre os grupos, mas foi significante para ambos comparando o antes e o depois. Já os rolinhos modificados, no grupo A, aumentaram apresentando significância. No B reduziram apesar de não ter significância. Já na comparação entre grupos houve significância. 0 ganho de força abdominal em ambos os grupos é explicado pelos fatores neurais que influenciam na produção de força muscular por meio da maior eficiência no recrutamento neural, na excitabilidade dos motoneurônios e na ativação do sistema nervoso central, melhorando o sincronismo das unidades motoras e inibindo o órgão tendinoso de Golgi $(26,29)$.

Embora o protocolo de fortalecimento abdominal aplicado tenha sido idêntico em ambos os grupos, o que associou a contração perineal e a abdominal teve melhores ganhos na força e na resistência muscular. Tal fato é explicado pelo sinergismo entre a MAP e a ação do transverso abdominal, cuja função foi potencializada pela associação da contração perineal ao fortalecimento abdominal, resultando, além do aumento da força perineal, em um aumento de força abdominal mais evidente do que no grupo que não teve tal associação (30-32). Também se somam a esta pesquisa estudos dos músculos abdominais profundos, com eletromiografia de agulha, os quais demonstraram que o transverso do abdome é o principal músculo abdominal responsável por gerar a PIA. Graças à orientação horizontal de suas fibras, a contração desse músculo resulta em diminuição da circunferência abdominal, o que leva ao aumento da tensão da fáscia tóraco-lombar e, por fim, ao aumento da PIA $(33,34)$. No entanto, alguns autores contradizem esse achado e relatam parecer que uma contração do transverso abdominal pode não elevar o assoalho pélvico (para apoiar os órgãos) em todas as mulheres (35). Portanto, o papel dos músculos transversos abdominais em um protocolo de fortalecimento dos músculos do assoalho pélvico ainda não é bem compreendido (36).

\section{Conclusão}

De acordo com os resultados apresentados neste estudo foi possível concluir que o fortalecimento abdominal influencia na força e na função da musculatura do assoalho pélvico, visto que o grupo submetido ao treino de fortalecimento associando a contração dessa musculatura obteve resultados significativos se comparado ao outro grupo (teste dos cones e teste de resistência/pressão na perineometria), que apresentou piora na maioria das variáveis.

Portanto, sugere-se que a contração abdominal associada à contração perineal pode promover o aumento de força e função da MAP, além de potencializar o ganho de força na musculatura abdominal, fato comprovado pelo teste de descida de membros inferiores, provavelmente em razão da ação do músculo transverso abdominal, que sinergicamente atua na contração perineal.

Foi observado que a mulheres praticantes de atividades físicas nunca foram orientadas, durante o treino abdominal, para associar a contração perineal, favorecendo dessa forma o aparecimento da incontinência urinária, como sugerem os resultados deste estudo.

A continência urinária na mulher é garantida pelo assoalho pélvico, portanto, este deve ser trabalhado nos treinamentos, nas academias e nos clubes, como qualquer outro músculo do corpo. Assim sendo, os profissionais que trabalham com atividades físicas devem ser preparados e informados a respeito das consequências do destreino dessa musculatura.

As mulheres procuram a academia visando ao bem-estar geral, tornando-se desse modo necessária a orientação de contração perineal adequada pelos profissionais que aplicam um protocolo de fortaleci- 
mento muscular, uma vez que a maioria delas não tem noção corporal e, se não forem orientadas holisticamente, sua qualidade de vida poderá sofrer prejuízo.

Dessa forma, sugere-se que estudos futuros sejam realizados com um número maior de indivíduos e com maior controle de variáveis, incluindo na avaliação um equipamento de eletromiografia que permita mensurar com maior precisão os ganhos alcançados com o protocolo de fortalecimento e observar o sinergismo da musculatura abdominal nos resultados, para que dessa forma seja possível determinar um protocolo com melhores resultados clínicos.

\section{Agradecimentos}

Os autores agradecem à empresa Quark por ter cedido gentilmente o equipamento de miofeedback para o desenvolvimento dessa pesquisa, bem como à empresa Profilática por ter cedido o material para a desinfecção do equipamento.

\section{Referências}

1. Nagib ABL, Guirro ECO, Palauro VA, Guirro RRJ. Avaliação da sinergia da musculatura abdomino-pélvica em nulíparas com eletromiografia e biofeedback perineal. Rev Bras Ginecol Obstet. 2005;27(4):210-5.

2. Caetano AS, Tavares MCGCF, Lopes MHBM, Poloni RL. Influência da atividade física na qualidade de vida e auto-imagem de mulheres incontinentes. Rev Bras Med Esporte. 2009;15(2):93-7.

3. Araújo MP, Oliveira E, Zucchi EVM, Trevisani VFM, Girão MJBC, Sartori MGF. Relação entre incontinência urinária em mulheres atletas corredoras de longa distância e distúrbio alimentar. Rev Assoc Med Bras. [online]. 2008;54(2):146-9.

4. Polden M, Mantle J. Fisioterapia em obstetrícia e ginecologia. 2a ed. São Paulo: Santos; 2000.

5. Capelini MVMA. Tratamento da incontinência urinária de esforço com biofeedback: análise objetiva e impacto sobre a qualidade de vida [dissertação]. São Paulo: Unicamp; 2005.

6. Chiarapa TR, Cacho DP, Alves AFD. Incontinência urinária feminina: assistência fisioterapêutica e multidisciplinar. São Paulo: Livraria Médica Paulista; 2007.
7. Moreira SFS, Girão MJBC, Sartori MGF, Baracat EC, Lima GR. Mobilidade do colo vesical e avaliação funcional do assoalho pélvico em mulheres continentes e com incontinência urinária de esforço, consoante o estado hormonal. Rev Bras Ginecol Obstet. 2002;24(6):365-70.

8. Moreno AL. Fisioterapia em uroginecologia. São Paulo: Manole; 2004.

9. Kendall FP, McCreary EK, Provance PG, Rodgers MM, Romani WA. Músculos: provas e funções. 5a ed. São Paulo: Manole; 2007.

10. Palmer ML, Epler ME. Fundamentos das técnicas de avaliação musculoesquelética. 2a ed. Rio de Janeiro: Guanabara Koogan; 2000.

11. Brooks D. O livro completo para o treinamento personalizado. São Paulo: Phorte; 2008.

12. Honório MO, Santos SMA. Incontinência urinária e envelhecimento: impacto no cotidiano e na qualidade de vida. Rev Bras Enferm. 2009;62(1):51-6.

13. Higal R, Lopes MHBM, Reis MJ. Fatores de risco para incontinência urinária na mulher. Rev Esc Enferm USP. 2008;42(1):187-92.

14. Matheus LM, Mazzari CF, Mesquita RA, Oliveira J. Influência dos exercícios perineais e dos cones vaginais, associados à correção postural, no tratamento da incontinência urinária feminina. Rev Bras Fisioter. 2006;10(4):387-92.

15. Dreher DZ, Berlezi EM, Strassburger SZ, Ammar MZE. O fortalecimento do assoalho pélvico com cones vaginais: programa de atendimento domiciliar. Scientia Med. 2009;19(1):43-9.

16. Oliveira JMS, Salgado LBG, Schmitt ACB, Rosa LCL. Correlação entre sintomas urinários e qualidade de vida em mulheres com incontinência urinária. Fisioter Pesq. 2007;14(3):12-7.

17. Araujo AD, Arruda MBA, Jammal MP, Guimarães EL. Estudo da prevalência de incontinência urinária e seu impacto na qualidade de vida de mulheres - Projeto "Espaço Mulher 2008". Fisioter Especialid. 2008; 2(2):42-6.

18. Silveira EA, Kac G, Barbosa LB. Prevalência e fatores associados à obesidade em idosos residentes em Pelotas, Rio Grande do Sul, Brasil: classificação da obesidade segundo dois pontos de corte do índice de massa corporal. Cad Saúde Pública. 2009;25(7):1569-77. 
19. Bo K, Wesnes SL, Hunskaar S, Rortveit G. The effect of urinary incontinence status during pregnancy and delivery mode on incontinence postpartum. BJOG. 2009,116(5):700-7.

20. Fozzatti MCM, Palma P, Herrmann V, Dambros M. Impacto da reeducação postural global no tratamento da incontinência urinária de esforço feminina. Rev Assoc Med Bras. 2008;54(1):17-22.

21. Marques LSF, Freitas PAC. A cinesioterapia como tratamento da incontinência urinária na unidade básica de saúde. Fisioter Mov. 2005;18(4):63-7.

22. Moreira ECH, Yasuda EK, Kimura FR. Tratamento cirúrgico e conservador da incontinência urinária de esforço. Fisioter Mov. 2001;13(2):9-14.

23. Barros JD, Lucena ACT, Anselmo CWSF. Incontinência urinária de esforço em atletas do sexo feminino: uma revisão da literatura. An Fac Med Univ Fed Pernamb. 2007;52(2):173-80.

24. Parente LC, Henriques SHFC. Fisioterapia na incontinência urinária no pós-parto. Fisioter Brasil. 2007; 8(4):288-93.

25. Robergs RA, Roberts S. Princípios fundamentais de fisiologia do exercício: para aptidão, desempenho e saúde. São Paulo: Phorte; 2002.

26. McArdle WS, Katch FI, Katch VL. Fisiologia do exercício: energia, nutrição e desempenho humano. 6a ed. Rio de Janeiro: Guanabara Koogan; 2008.

27. Bucci M, Vinagre EC, Campos GER, Curi R, PithonCuri TC. Efeitos do treinamento concomitante hipertrofia e endurance no músculo esquelético. Rev Bras Ciênc Mov. 2005;13(1):17-28.

28. Lamim SEM, Santos MF, Silva MM, Rotta AL, Pudo MA. Avaliação do grau de força muscular do períneo em mulheres praticantes de musculação. Fisioter Brasil. 2006;7(4):268-71.
29. Wilmore JH, Costill DL. Fisiologia do esporte e do exercício. 2a ed. São Paulo: Manole; 2001.

30. Lemos AM, Feijó LA. A biomecânica do transverso abdominal e suas múltiplas funções. Fisioter Brasil. 2005;6(6):66-70.

31. Gouveia KMC, Gouveia EC. O músculo transverso abdominal e sua função de estabilização da coluna lombar. Fisioter Mov. 2008;21(3):45-50.

32. Neumann P, Gill V. Pelvic floor and abdominal muscle interaction: EMG activity and intra-abdominal pressure. Int Urogynecol J Pelvic Floor Dysfunct. 2002; 13(2):125-32.

33. Salmela LFT, Sakamoto ACL, Siqueira FB. Mecanismos de estabilização da coluna lombar: uma revisão da literatura. Fisioter Mov. 2004;17(4):51-7.

34. Sapsford RR, Hodges PW. Contraction of the pelvic floor muscles during abdominal maneuvers. Arch Phys Med Rehabil. 2001;82(8):1081-8.

35. Bø K, Sherburn M, Allen T. Transabdominal ultrasound measurement of pelvic floor muscle activity when activated directly or via a transversus abdominis muscle contraction. Neurourol Urodyn. 2003; 22(6):582-8.

36. Thomas KL, Sam D, Anand P. Pelvic floor muscle training in combination with another therapy compared with the other therapy alone for urinary incontinence in women (Protocol for a Cochrane Review). The Cochrane Library. 2010;(10).

Recebido: 17/05/2010

Received: 05/17/2010

Aprovado: 27/11/2010

Approved: $11 / 27 / 2010$ 


\section{Apêndice - Protocolo de fortalecimento abdominal}

1) Reto-abdominal

Posição: sujeito em DD sobre um colchonete, com MMII em flexão e pés apoiados no solo, MMSS de acordo com o período do treinamento.

Tabela 1 - Distribuição do tempo do exercício reto abdominal - fibras superiores

\begin{tabular}{lc}
\hline Técnica & Tempo(s) \\
\hline Manter a posição inicial, inspirando. & 2 \\
Ao expirar, contrair os músculos do períneo e os músculos abdominais, elevando a caixa & 3 \\
torácica, uma vértebra por vez, em direção à pelve. & 1 \\
Na amplitude máxima sustentar a posição, expirando com contração do assoalho pélvico. & 3 \\
Voltar, lentamente, à posição inicial. & \\
\hline
\end{tabular}

Obs.: 0 grupo B não recebeu a orientação da contração do períneo.

Tabela 2 - Posição dos MMSS de acordo com o período de treinamento

\begin{tabular}{lc}
\hline Posição dos MMSS & Dias de treino \\
\hline Paralelos ao tronco & $1^{0}$ ao $6^{0}$ \\
Sobre o tórax & $7^{0}$ ao $12^{\circ}$ \\
Atrás da cabeça & $13^{\circ}$ ao $18^{\circ}$ \\
\hline
\end{tabular}

Fonte: Adaptado de Kendall, McCreary e Provance (1995).

2) Abdominal - fibras inferiores

Posição: sujeito em DD sobre um colchonete, MMII paralelos ao solo, MMSS sob o tórax. Flexionar o quadril a $90^{\circ}$, manter os joelhos estendidos e a pelve retrovertida. Retornar os MMII até a angulação determinada para o período do treinamento.

Tabela 3 - Angulação da flexão de quadril de acordo com o período de treinamento

\begin{tabular}{lc}
\hline Ângulo & Dias de treino \\
\hline $75^{\circ}$ & $1^{0}$ ao $6^{\circ}$ \\
$45^{\circ}$ & $7^{0}$ ao $12^{\circ}$ \\
$0^{\circ}$ & $13^{\circ}$ ao $18^{\circ}$ \\
\hline
\end{tabular}

Fonte: Adaptado de Kendall, McCreary e Provance (1995). 
Tabela 4 - Distribuição do tempo do exercício reto-abdominal - infra-abdominal

\begin{tabular}{lc}
\hline Técnica & Tempo(s) \\
\hline $\begin{array}{l}\text { Abaixar lentamente os MMIl até a angulação determinada de acordo com o período de } \\
\text { treinamento. }\end{array}$ & 3 \\
Sustentar a posição, inspirando. & 1 \\
Ao expirar, contrair os músculos do períneo e os músculos abdominais, elevando os MMII. & 3 \\
Na amplitude máxima sustentar a posição, expirando e contraindo o períneo. & 1 \\
\hline
\end{tabular}

Obs.: o grupo B não recebeu a orientação da contração do períneo.

3) Oblíquos internos e externos do abdômen do reto-abdominal

Posição: sujeito em DD, com MMII em flexão e pés apoiados no solo, MMSS de acordo com o período do treinamento (Tabela 5).

Tabela 5 - Distribuição do tempo do exercício oblíquo do abdômen

\begin{tabular}{lc}
\hline Técnica & Tempo(s) \\
\hline Na posição inicial, inspirar. & 2 \\
Ao expirar, contrair a musculatura do assoalho pélvico e os músculos abdominais oblíquos, & 3 \\
flexionando e rodando o tronco. & 1 \\
Na amplitude máxima sustentar a posição, expirando e contraindo o períneo. & 2 \\
Voltar, lentamente, à posição inicial. & \\
\hline
\end{tabular}

Obs.: 0 grupo B não recebeu a orientação da contração do períneo. 